\title{
A determinant of feline immunodeficiency virus involved in Crandell feline kidney cell tropism
}

\author{
Kees H.J. Siebelink $k^{\mathrm{a}}$, Jos A. Karlas ${ }^{\mathrm{a}}$, Guus F. Rimmelzwaan ${ }^{\mathrm{b}}$, \\ Albert D.M.E. Osterhaus ${ }^{\mathrm{a}, \mathrm{b}, *}$, Marnix L. Bosch ${ }^{\mathrm{a}}$ \\ a Laboratory of Immunobiology, National Institute of Public Health and Environmental Protection. \\ Antonie van Leeuwenhoeklaan 9, PO Box 1, 3720 BA Bilthoven. The Netherlands \\ 'Institute of Virology, Erasmus University Rotterdam, Dr. Molewaterplein 50, 30I5 GE Rotterdam,
}

The Netherlands

\begin{abstract}
Viral progeny of the molecular clone $19 \mathrm{k} 1$ of feline immunodeficiency virus (FIV) can infect feline T-cells but not Crandell feline kidney ( $\mathrm{CrFK}$ ) cells. In contrast, the biological isolate FIVAM6c, which was CrFK adapted by co-cultivation of FIV-AM6 infected thymocytes with CrFK cells, can infect both thymocytes and CrFK cells. The envelope gene of FIV-AM6c was amplified by polymerase chain reaction using DNA from infected CrFK cells, and subsequently cloned and sequenced. To map viral determinants of CrFK cell tropism, chimeric viruses with a $19 \mathrm{k} 1$ background containing envelope gene fragments of FIV-AM6c were constructed. CrFK cells were transfected with DNA of these chimeric clones and co-cultivated with thymocytes. After 3 days the CrFK cells and the thymocytes were cultured separately. FIV antigen could be detected in most of the thymocyte cultures within 14 days and in one of the CrFK cultures after 52 days. The resulting virus from this CrFK culture can infect both CrFK cells and thymocytes. The results of this study indicate that the envelope region contains determinants of CrFK tropism. The delay in replication indicates that also determinants other than those identified here are involved in CrFK cell tropism. More chimeric clones are being studied at present to map these determinants.
\end{abstract}

\section{Abbreviations}

Con A, Concanavalin A; cpe, cytopathic effect; CrFK cells, Crandell feline kidney cells; FeSFV, feline syncytium forming virus; FIV, feline immunodeficiency virus; HIV-1, human immunodeficiency virus type 1; PBMCs, peripheral blood mononuclear cells; PCR, polymerase chain reaction; SPF, specified pathogen-free.

\footnotetext{
* Corresponding author at: Institute of Virology, Erasmus University Rotterdam, Dr. Molewaterplein 50, 3015 GE Rotterdam, The Netherlands. Tel: (31) 10-4088066; Fax: (31) 10-4365145.
} 


\section{Introduction}

Feline immunodeficiency virus (FIV), like the other lentiviruses, displays a high degree of sequence variation in the envelope gene. Most of this variation maps to the so-called variable regions (Phillips et al., 1990; Pancino et al., 1993). In other lentivirus systems, this genetic variation often has biological consequences, e.g. with regard to cytopathic potential and cell tropism (Cheng-Mayer et al., 1990). The variable region number 3 (V3) of the human immunodeficiency virus type 1 (HIV-1) envelope, which contains the principal neutralizing determinant, also contains the primary determinants for both cell tropism and for syncytium inducing capacity (Grimaila et al., 1992), although variation in both these parameters has also been mapped to regions other than V3 (Thali et al., 1991; Andeweg et al., 1993).

FIV can infect a variety of cell types, like CD4 and CD8 positive T-cells, macrophages and astrocytes (Brunner and Pedersen, 1989; Dow et al., 1990; Brown et al., 1991). Furthermore some strains of FIV can infect Crandell feline kidney cells ( $\mathrm{CrFK}$ cells) upon adaptation in vitro (Phillips et al., 1990). Infection of CrFK cells with FIV is widely used in virus neutralization assays. Using this system a variety of FIV neutralizing antibodies have been described. Most sera that neutralize FIV infection of $\mathrm{CrFK}$ cells tend to be broadly reactive, i.e. they all neutralize the different CrFK-adapted FIV strains, independent of the viral isolate against which they were initially raised (Tozzini et al., 1993). Such FIV neutralizing antibodies have also been generated in rabbits and mice using a synthetic peptide corresponding to the V3 region of the FIV envelope (Lombardi et al., 1993). This peptide also corresponds to an immunodominant epitope in FIV infected cats (Avrameas et al., 1992), which suggests that antibodies to this region contribute to the FIV-neutralizing activity in the sera of these cats.

Recently we have described a virus neutralization assay for FIV using activated T cells, one of the natural target cells for the virus (Siebelink et al., 1993). With this assay we have only been able to demonstrate genotypic-specific virus neutralization: singlc point mutations, either induced in vitro, or naturally occurring, completely abolished virus neutralization by a polyclonal serum. No evidence for broad cross-neutralization of FIV was found. These differences in virus neutralization between the two systems, led us to investigate the molecular basis for the adaptation of FIV to CrFK cells. Presently nothing is known of the relationship between genetic variation of FIV and cell tropism. Information regarding this issue may help us to understand the requirements for the infection of different cell types by FIV and, at the same time, may reveal the important biological aspects involved in virus neutralization in both systems.

In order to determine whether the adaptation of FIV to CrFK cells is dependent on virus entry we have exchanged parts of the surface glycoprotein of FIV molecular clone 19k1 (which cannot replicate in CrFK cells) with the homologous fragments of the CrFK-adapted virus strain FIV-AM6c. We examined these chimeric clones for the potential to replicate in feline thymocytes and/or CrFK cells.

\section{Materials and methods}

\subsection{Cells and virus}

Peripheral blood mononuclear cells (PBMCs) were derived from heparinized blood of a 2-year-old specified pathogen-free (SPF) cat by Ficoll density gradient centrifugation. 
Thymocytes were derived by homogenizing the thymus of an 8-week-old SPF cat. PBMCs and thymocytes were washed twice and frozen at $-135^{\circ} \mathrm{C}$ in aliquots. Before use the cells were thawed and stimulated with Concanavalin A (Con A) $\left(5 \mu \mathrm{g} \mathrm{ml}^{-1}\right)$ in CM (RPMI1640 supplemented with penicillin ( $\left.100 \mathrm{IU} \mathrm{ml}^{-1}\right)$, streptomycin $\left(100 \mu \mathrm{g} \mathrm{ml}^{-1}\right.$ ), L-glutamine $(2 \mathrm{mM})$ and $\beta$-mercaptoethanol $\left(2 \times 10^{-5} \mathrm{M}\right)$ and $10 \%$ fetal calf serum). After 3 days the cells were washed and cultured in CM supplemented with IL-2 (100 IU ml $\left.{ }^{-1}\right)$.

An FIV susceptible clone of the CrFK cell line was obtained from N. Pedersen (Yamamoto et al., 1988) and cultured as described previously (Siebelink et al., 1992).

Replication competent molecular clone $19 \mathrm{k} 1$ was obtained directly from bone marrow cells of a naturally FIV infected cat as described previously (Siebelink et al., 1992).

FIV was isolated from PBMCs from 11 naturally FIV infected cats. To this end the PBMCs of these cats were stimulated with Con A. After 3 days the cells were washed and co-cultured with Con A and IL-2 stimulated PBMCs from a SPF cat in CM supplemented with IL-2 ( $\left.100 \mathrm{IU} \mathrm{ml}^{-1}\right)$. The culture supernatant was tested weekly for the presence of FIV antigen by ELISA ( see below). When FIV antigen was detected subconfluent monolayers of CrFK cells were co-cultivated for 1 week with $10^{6}$ infected PBMCs. The CrFK cells were washed, trypsinized and subcultured at a split ratio of 1:5 weekly. The culture supernatants were tested weekly in an FIV antigen ELISA. One of the FIV isolates which was adapted to replicate in CrFK cells was designated FIV-AM6c and was used in this study.

\subsection{FIV antigen ELISA}

Culture supernatants were tested in an ELISA for the presence of FIV antigen as described previously (Siebelink et al., 1989).

\subsection{Generation of chimeric clones}

The envelope gene of FIV-AM6c was amplified by polymerase chain reaction (PCR) ds described previously (Siebelink et al., 1993). The env gene was cloned into pUC19 and the sequence was determined by the dideoxynucleotide chain termination reaction. The nucleotide sequence was compared with the sequence of the envelope gene of molecular clone $19 \mathrm{k} 1$. To generate chimeric clones four conserved restriction sites were used to exchange parts of the envelope gene of $19 \mathrm{k} 1$ with corresponding parts of FIV-AM6c (Fig. 1). Three chimeric clones were generated: 19k1PBAM6 (19k1 which contain the $144 \mathrm{bp}$ PfIM-BsaI fragment of FIV-AM6c), 19k1BNAM6 (19k1 which contain the 343 bp BsalNsiI fragment of FIV-AM6c) and 19k1NKAM6 (19k1 which contain the 248 bp NsilKpnI fragment of FIV-AM6c).

\subsection{Transfection}

Five micrograms DNA of the molecular clone 19k1 and the chimeric clones 19k1PBAM6, 19k1 BNAM6 and 19k1NKAM6 were transfected into CrFK cells using the cationic lipid DOTMA (lipofectin; Bethesda Research Laboratories Inc.) according to the protocol of the manufacturer. After $24 \mathrm{~h}$ the transfected cells were cocultivated with Con A- and IL-2- 


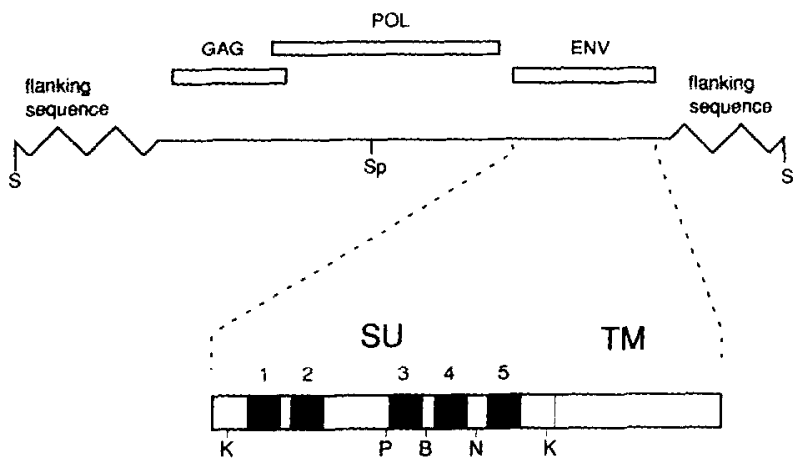

$19 \mathrm{k} 1$

AM6c

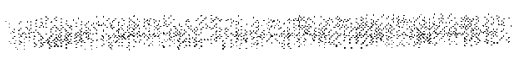

19k1PBAM6

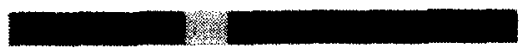

19k1BNAM6

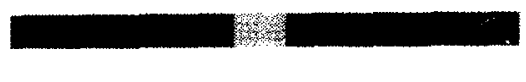

19k1NKAM6

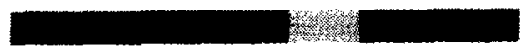

Fig. 1. Schematic representation of the envelope genes of molecular clone $19 \mathrm{k} 1$ and the chimeric clones of $19 \mathrm{k} 1$ which contain the hypervariable regions 3,4 or 5 of the CrFK adapted isolate FIV AM6c. The uppermost bars represent the major open reading frames gag, pol and env. The black boxes represent the hypervariable regions in the envelope protein. The patterns are corresponding to the parental clones.

stimulated feline thymocytes. After 3 days the $\mathrm{CrFK}$ cells and thymocytes were cultured separately. The culture supernatants were monitored for the presence of FIV antigen by ELISA weekly. When FIV antigen could be detected, the culture supernatant was collected, filtered through a $220 \mathrm{~nm}$-pore-size filter and stored in aliquots.

\section{Results}

\subsection{Adaptation of FIV to replicate in $\mathrm{CrFK}$ cells}

Con A and IL-2 stimulated PBMCs were infected with 11 FIV isolates in separate cultures. Within 7 days FIV antigen could be detected in the supernatants of all cultures by ELISA (Table 1). The PBMCs were then co-cultured with CrFK cells. In four cultures a cytopathic effect (cpe) typical for feline syncytium forming virus (FeSFV) was observed in the CrFK cell culture (not shown). These cultures were discarded. FIV antigen could only be demonstrated in the CrFK cell culture infected with FIV-AM4 and FIV-AM6 28 days post infection (Table 1). Viral progeny of molecular clone $19 \mathrm{k} 1$ could not be adapted to replicate in $\mathrm{CrFK}$ cells using this procedure (not shown). 
Table 1

FIV antigen detection in culture supernatants of PBMCs and $\mathrm{CrFK}$ cells

\begin{tabular}{lll}
\hline Isolate & \multicolumn{2}{l}{ FIV antigen in supernatant of } \\
\cline { 2 - 3 } & PBMCs & CrFK cells \\
\hline FIV-AM1 & + & - \\
FIV-AM2 & + & - \\
FIV-AM3 & + & NT \\
FIV-AM4 & + & + \\
FIV-AM5 & + & - \\
FIV-AM6 & + & + \\
FIV-AM7 & + & NT \\
FIV-AM8 & + & - \\
FIV-AM9 & + & NT \\
FIV-AM10 & + & NT \\
FIV-AM19 & + & - \\
\hline
\end{tabular}

NT, not tested.

\subsection{Transfection of chimeric clones}

Upon transfection with DNA of the molecular clone $19 \mathrm{kl}$ and the chimeric clones 19k1PBAM6, 19k1BNAM6 and 19k1NKAM6 the CrFK cells were cocultivated with thymocytes. After $72 \mathrm{~h}$ the $\mathrm{CrFK}$ cells and thymocytes were cultured separately and monitored for the presence of FIV antigen in the culture supernatant. Within 3 weeks post transfection FIV antigen was detected in the supernatant of all the thymocyte cultures (Fig. 2a). Only in the culture supernatant of the CrFK cells, which were transfected with 19k1PBAM6 FIV antigen could be detected 52 days post transfection whereas no antigen could be detected in the culture supernatant of the CrFK cells, which were transfected with 19k1, 19k IBNAM6 or 19k1NKAM6 (Fig. 2b).

The culture supernatant of the CrFK cells, which was found positive in the FIV antigen ELISA was collected 52 days post transfection, filtered and designated FIV-19k1PBAM6c. CrFK cells were infected with FIV-19k1PBAM6c or FIV-AM6c and monitored at regular intervals for FIV antigen production in the culture supernatant. Within 11 days FIV antigen could be detected in the supernatant of both cultures (Fig. 3). These results show that chimeric clone 19k1PBAM6c is adapted to infect and replicate in CrFK cells with the same kinetics as FIV-AM6c.

\subsection{Sequence analysis}

To minimize additional mutations by long-term culture, 4 days after infection with 19k1PBAM6c, CrFK cells were lysed and the FIV envelope gene was amplified by PCR and cloned into pUC19. The surface protein was sequenced and compared with the sequence of $19 \mathrm{k} 1$. The $19 \mathrm{k} 1$ PBAM $6 \mathrm{c}$ sequence differs at five positions from the $19 \mathrm{k} 1$ env sequence (Fig. 4). Four of these, three situated in HV-3 and one between HV-3 and HV-4, are the result of the exchange of the 144 bp PflMI-BsaI fragment of FIV-AM6c. An additional G 

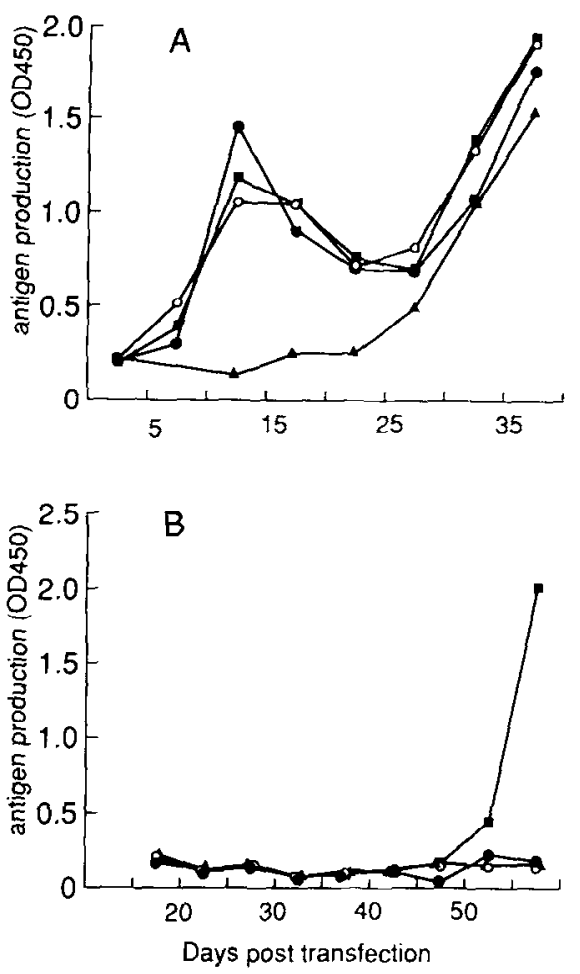

Fig. 2. FIV antigen production in culture supernatant of PBMCs (A) and CrFK cells (B) after transfection with

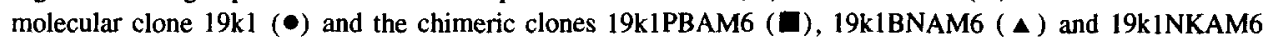
(O).

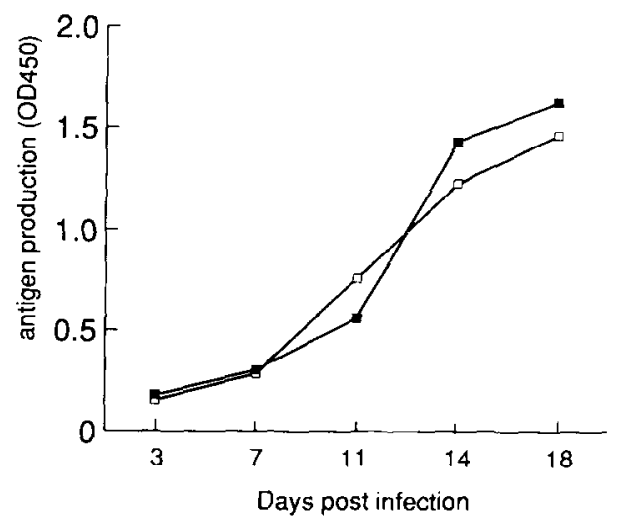

Fig. 3. FIV antigen production in culture supernatant of CrFK cells infected with FIV-AM6c ( $\square$ ) and FIV19k1PBAM6c (西). 


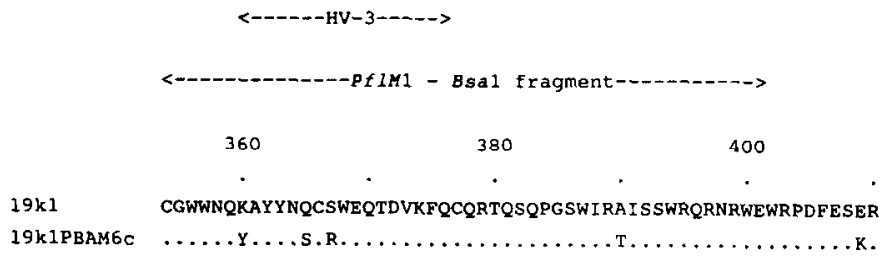

Fig. 4. Amino acid sequence of 19k1 and 19k1 PBAM6c from amino acid position 354 to 410 . The 144 bp PflMI$B s a l$ fragment and the HV-3 region is indicated at the top.

to A mutation at nucleotide position 1225 was observed, which causes an amino acid substitution mutation of glutamic acid to lysine at position 409 .

\section{Discussion}

A limited number of all FIV isolates known to date replicate in CrFK cells. These cells are widely used in FIV-neutralization assays which makes elucidation of the molecular basis for CrFK-adaptation of FIV an important research goal. This importance is underlined by the fact that using these cells predominantly broadly neutralizing antibodies are detected, which contrast with the results using $T$ cells, one of the natural target cells for FIV.

The viral progeny of FIV molecular clone 19k1 cannot infect CrFK cells, and we have never been able to adapt FIV 19k1 to grow in these cells even after prolonged periods of cocultivation of CrFK cells with FIV 19k1 infected T cells (up to 3 months). Transfer of an envelope fragment of the CrFK-adapted FIV-AM6c isolate to $19 \mathrm{k} 1$ has now allowed us to adapt $19 \mathrm{kl}$ to $\mathrm{CrFK}$ cells after 52 days. This envelope fragment contains the variable V3 region; no other envelope fragments have yielded similar results. The resulting adapted virus is now able to infect CrFK cells and does not demonstrate the 52-day delay observed in the adaptation process. It is therefore likely that the fully adapted virus has accumulated other mutations, in the envelope gene or elsewhere, that have now converted the phenotype of the virus to the fully adapted form. One of such changes, just $3^{\prime}$ of the originally exchanged V3 region is discussed below. Preliminary experiments indicate that this single mutation is not by itself sufficient for adaptation (in preparation). We currently investigate the contribution of other envelope regions and of the other viral genes to $\mathrm{CrFK}$ adaptation.

Other lentiviruses, like HIV-1, also display a high degree of genetic variation in the envelope gene. Mapping experiments have demonstrated that the highly variable V3 region of the HIV-1 envelope contains an important determinant for cytopathicity and cell tropism, as well as the principal neutralization domain (Grimaila et al., 1992). In our experiments we could only adapt FIV $19 \mathrm{k} 1$ to replicate in CrFK cells when we replaced the V3 region of $19 \mathrm{k} 1$ with the envelope $\mathrm{V} 3$ region of the CrFK-adapted virus FTV-AM6c, suggesting that here also the $\mathrm{V} 3$ region plays a role in determining cell tropism. This parallel between the functional roles of the V3 regions of HIV-1 and FIV can be extended when we take into account that a synthetic V3 peptide of both of these viruses can elicit virus neutralizing antibodies. Interestingly in the FIV system these antibodies will only neutralize FIV infection of CrFK cells, and not of primary $\mathrm{T}$ cells. Understanding the requirements for FIV to productively infect $\mathrm{CrFK}$ cells may elucidate the basis for virus neutralization in both 
systems. This information will prove useful in the rational design of virus vaccines that aim at inducing high titers of broadly reactive virus neutralizing antibodies, both for FIV and for HIV-1.

Since we have determined only the sequence of the gene encoding the surface glycoprotein in this study, we cannot exclude that other mechanisms play a role in the acquisition of CrFK cell tropism by FIV. Our present studies focus on the potential involvement of the gag, pol and regulatory genes of FIV in this phenomenon.

\section{Acknowledgements}

We thank C. Kruyssen for preparing the manuscript and W. Puyk for performing the pepscan analysis. This work was supported by the Advisory Council on Health Research (RGO No. 88-90/89028) and the concerted action on feline AIDS of the EC.

\section{References}

Andeweg, A.C., Leeflang, P., Osterhaus, A.D.M.E. and Bosch, M.L., 1993. Both the V2 and V3 regions of the human immunodeficiency virus type 1 surface glycoprotein functionally interact with other envelope regions in syncytium formation. J. Virol., 67: 3232-3239.

Avrameas, A., Guillet, J.G., Chouchane, L., Moraillon, A., Sonigo, P. and Strosberg, A.D., 1992. Localisation of three epitopes of the env protein of feline immunodeficiency virus. Mol. Immunol., 29: 565-572

Brown, W.C., Bissey, L., Logan, K.S., Pedersen, N.C., Elder, J.H. and Collison, E.W., 1991. Feline immunodeficiency virus infects both CD4 + and CD8 + T lymphocytes. J. Virol., 65: 3359-3364.

Brunner, D. and Pedersen N.C., 1989. Infection of peritoneal macrophages in vitro and in vivo with feline immunodeficiency virus. J. Virol., 63: 5483-5488.

Cheng-Mayer, C., Quiroga, M., Tung, J.W., Dina, D. and Levy, J.A., 1990. Viral determinants of human immunodeficiency virus type $1 \mathrm{~T}$-cell or macrophage tropism, cytopathogenicity, and CD4 antigen modulation. J. Virol., 64: 4390-4398.

Dow, S.W., Poss, M.L. and Hoover, E.W., 1990. Feline immunodeficiency virus: a neurotropic virus. J. AIDS, 3 : $658-668$.

Grimaila, R.J., Fuller, B.A., Rennert, P.D., Nelson, M.B., Hammarskjöld, M., Potts, B., Murray, M., Putney, S.D. and Gray, G., 1992. Mutations in the principal neutralization determinant of human immunodeficiency virus type 1 affect syncytium formation, virus infectivity, growth kinetics, and neutralization. J. Virol., 66: 18751883.

Lombardi, S., Garzelli, C., LaRosa, C., Zaccaro, L., Specter, S., Malvaldi, G., Tozzini, F., Esposito, F. and Bendinelli, M., 1993. Identification of a linear neutralization site within the third variable region of the feline immunodeficiency virus envelope. J. Virol., 67: 4742-4749.

Pancino, G., Chappey, C., Saurin, W. and Sonigo, P., 1993. B epitopes and selection pressures in feline immunodeficiency virus envelope glycoproteins. J. Virol., 67: 664-672.

Phillips, T.R., Talbott, R.L., Lamont, C., Muir, S., Lovelace, K. and Elder, J.H., 1990. Comparison of two host cell range variants of feline immunodeficiency virus. J. Virol., 64: 4605-4613.

Siebelink, C.H.J., Windrich, R.W., Rimmelzwaan, G.F., Weijer, K., Van Herwijnen, R., Knell, P., Egberink, H.F., Bosch, M.L. and Osterhaus, A.D.M.E., 1989. An enzyme linked immunosorbent assay (ELISA) for the detection of feline immunodeficiency virus (FIV) antigen in cell culture and FIV specific antibodies in feline serum. Dev. Biol. Stand., 72: 189-196.

Siebelink, K.H.J., Chu, I., Rimmelzwaan, G.F., Weijer, K., Osterhaus A.D.M.E. and Bosch., M.L., 1992. Isolation and partial charactcrization of infectious molecular clones of feline immunodeficiency virus obtained directly from bone marrow DNA of a naturally infected cat. J. Virol., 66: 1091-1097. 
Siebelink, K.H.J., Rimmelzwaan, G.F., Bosch M.L., Meloen, R.H. and Osterhaus, A.D.M.E., 1993. A single amino acid substitution in hypervariable region 5 of the envelope protein of feline immunodeficiency virus allows escape from virus neutralization. J. Virol., 67: 2202-2208.

Thali, M., Olshevsky, U., Furman, G., Gabuzda, D., Posner, M. and Sodroski, J., 1991. Characterization of a discontinuous human immunodeficiency virus type $1 \mathrm{gp} 120$ epitope recognized by a broadly reactive neutralizing human monoclonal antibody. J. Virol., 65: 6188-6193.

Tozzini, F., Matteucci, D., Bandecchi, P., Baldinotti, F., Siebelink, K., Osterhaus, A. and Bendinelli, M., 1993. Neutralizing antibodies in cats infected with feline immunodeficiency virus. J. Clin. Microbiol., 31: 16261629.

Yamamoto, J.K., Sparger, E., Ho, E.W., Andersen, P.R., O'Connor, T.P., Mandell, C.P., Lowenstine, L., Munn, R. and Pedersen, N.C., 1988. Pathogenesis of experinentally induced feline inmunodeficiency virus infection in cats. Am. J. Vet. Res., 49: 1246-1258. 